\title{
Diagnóstico de tuberculosis en el marco de la evaluación periódica ocupacional: observaciones a la actual normativa peruana
}

\section{Diagnosis of tuberculosis in the context of occupational periodic evaluation: observactions to the current Peruvian regulations}

\author{
Erik J. Jhonston ${ }^{1 . a}$, Christoper A. Alarcon-Ruiz ${ }^{2, b}$, Armando Miñan-Tapiaa,b ${ }^{3, \text { Alvaro Taype-Rondan }}{ }^{4, c}$ \\ ${ }^{1}$ Unidad de Seguridad y Salud Ocupacional y Medio Ambiente, Hospital Edgardo Rebagliati Martins, Lima, Perú. \\ ${ }^{2}$ Facultad de Medicina, Universidad Ricardo Palma, Lima, Perú. \\ ${ }^{3}$ Escuela Profesional de Medicina Humana, Universidad Privada de Tacna, Tacna, Perú. \\ ${ }^{4}$ Universidad San Ignacio de Loyola, Lima, Perú. \\ a Médico Residente de la especialidad de Medicina ocupacional y medio ambiente. \\ ${ }^{\mathrm{b}}$ Estudiante de medicina. \\ ${ }^{\circ}$ Médico cirujano.
}

\begin{abstract}
Correspondencia Armando Miñan-Tapia Teléf. (+51) 970976330 Direcc. Calle Santa Ana 1933 Centro Poblado Natividad, Tacna. Perúarluminan@gmail.com

Recibido: 10 de agosto 2017. Aceptado: 14 de agosto 2017.

Conflictos de interés: Los autores declaran no tener conflictos de interés.

Financiamiento: El presente artículo fue autofinanciado por los autores.

Contribuciones de los autores: EJJ, CAAR, AMT y ATR han participado en la concepción del artículo, la búsqueda bibliográfica, su redacción y aprobación de la versión final.
\end{abstract}

Citar como: Jhonston EJ, AlarconRuiz CA, Miñan-Tapia A, TaypeRondan A. Diagnóstico de tuberculosis en el marco de la evaluación periódica ocupacional: observaciones a la actual normativa peruana. An Fac med. 2017;78(4):461-2

DOl: http://dx.doi.org/10.15381/

anales.v78i4.14271
An Fac med. 2017;78(4):461-2 / http://dx.doi.org/10.15381/anales.v78i4.14271

Sr Editor, en Perú la Ley de Seguridad y Salud en el Trabajo vigente establece que el empleador es responsable de proteger la salud de sus trabajadores mediante actividades como los exámenes médico ocupacionales (EMO), que según la Resolución Ministerial (RM) 312-2011 del Ministerio de Salud (MINSA) son obligatorios para cada actividad económica ${ }^{(1)}$. Si bien esta RM ha implicado un avance en la salud ocupacional peruana, presenta ciertas falencias con respecto al tamizaje de tuberculosis, que podrían estar impidiendo un diagnóstico certero en la población vulnerable.

La RM solo obliga el tamizaje anual de tuberculosis para cuatro grupos ocupacionales: trabajadores de la salud (TS), personal de centros penitenciaros, segregadores y recicladores de residuos sólidos. Sin embargo, estudios nacionales y extranjeros han descrito otros grupos ocupacionales que también presentan un potencial riesgo de infección ocupacional por tuberculosis, como los que describimos en la Tabla 1. Aunque ciertamente pocos son los estudios nacionales sobre riesgo ocupacional de tuberculosis.

La RM solicita una radiografía de tórax para el diagnóstico de tuberculosis en el ámbito laboral, aunque algunos autores consideran que dicha prueba no sería de mucha utilidad para tal fin ${ }^{(2)}$, por lo que su requerimiento tal vez deba ser reevaluado. Además, la RM solicita una baciloscopía, la cual es rápida y poco costosa. Sin embargo, es una prueba con baja sensibilidad, y se ha encontrado que los TS tienen mayor porcentaje de casos de tuberculosis con baciloscopía negativa que la población general en Perú ${ }^{(3)}$, por lo que el uso de otras pruebas como el cultivo, la amplificación del ácido nucleico y la reacción en cadena de la polimerasa en tiempo real debería ser evaluado.

La RM propone una serie de EMO para TS, que lamentablemente no se están realizando en todos los establecimientos. Muestra de ello es que el $43,1 \%$ de médicos residentes peruanos que respondieron una encuesta a nivel nacional manifestaron que en su centro laboral no les realizan ni les exigen que se realicen ningún EMO ${ }^{(4)}$. Esta es una situación grave, dada la gran exposición de los TS a contagio por tuberculosis, como se evidencia en un reciente estudio realizado en el servicio de emergencias de un hospital de Lima, donde el $18,6 \%$ de los pacientes presentaron tuberculosis pulmonar activa ${ }^{(5)}$. 
Tabla 1: Grupos ocupacionales en potencial riesgo de tuberculosis.

\begin{tabular}{|c|c|c|}
\hline Grupo ocupacional & Justificación propuesta para estar en riesgo & Referencia \\
\hline Peones de la minería. & $\begin{array}{l}\text { Alto riesgo para desarrollar silicosis, la cual predispone } \\
\text { a infectarse o reactivar la tuberculosis. Además del } \\
\text { confinamiento en ambientes laborales poco ventilados. }\end{array}$ & $\begin{array}{l}\text { Stuckler D, et al. Mining and Risk of Tuberculosis in Sub- } \\
\text { Saharan Africa. Am J Public Health. 2011;101(3):524- } \\
\text { 530. }\end{array}$ \\
\hline $\begin{array}{l}\text { Personal de funerarias y } \\
\text { embalsamadores }\end{array}$ & $\begin{array}{l}\text { Contacto directo y manejo del cadáver infectado hace que } \\
\text { expulse el aire remanente de los pulmones conteniendo al } \\
\text { M. tuberculosis. }\end{array}$ & $\begin{array}{l}\text { Correia JC, et al. Assessing the survival of } \\
\text { Mycobacterium tuberculosis in unembalmed and } \\
\text { embalmed human remains. Clin Anat. 2014;27(3):304-7. }\end{array}$ \\
\hline $\begin{array}{l}\text { Conductores y cobradores de los } \\
\text { transportes públicos }\end{array}$ & $\begin{array}{l}\text { Constante contacto con personas en un ambiente sin } \\
\text { ventilación adecuada y en zonas con alta prevalencia de } \\
\text { infección por M. tuberculosis. }\end{array}$ & $\begin{array}{l}\text { Horna-Campos 0J, et al. Pulmonary tuberculosis infection } \\
\text { among workers in the informal public transport sector in } \\
\text { Lima, Peru. Occup Environ Med. 2011;68(2):163-5. }\end{array}$ \\
\hline $\begin{array}{l}\text { Personal de investigación en contacto } \\
\text { con pacientes con tuberculosis }\end{array}$ & $\begin{array}{l}\text { En el momento de la recolección de datos de pacientes } \\
\text { infectados por M. tuberculosis, se tiene un contacto } \\
\text { directo con ellos. }\end{array}$ & $\begin{array}{l}\text { Claassens MM, et al. Tuberculosis among community- } \\
\text { based health care researchers. Int J Tuberc Lung Dis. } \\
\text { 2010;14(12):1576-81 }\end{array}$ \\
\hline Personal de las fuerzas armadas & Expuestos a ambientes hacinados y con poca ventilación. & $\begin{array}{l}\text { O'Shea MK, Wilson D. Tuberculosis and the military. J R } \\
\text { Army Med Corps 2013;159(3):190-9 }\end{array}$ \\
\hline
\end{tabular}

Este incumplimiento de la norma en TS podría deberse a falencias en la supervisión de la misma, puesto que son varias las instituciones que comparten esta función. Si bien la realización de los EMO a los TS en general es supervisada por la Superintendencia Nacional de Fiscalización Laboral (SUNAFIL), distintas normas también delegan esta función a la Autoridad Nacional del Servicio Civil (SERVIR), a los Gobiernos Regionales, y a las Direcciones Regionales de Salud o de la que haga sus veces. Entidades que deberían coordinar sus acciones con SUNAFIL.
En resumen, creemos que las observaciones planteadas en la presente comunicación deberían tomarse en cuenta a la hora de realizar los EMO en Perú, e incluso ser tomados en consideración para modificar la RM 312-2011.

\section{REFERENCIAS BIBLIOGRÁFICAS}

1. Ministerio de Salud. Documento técnico: Protocolos de Exámenes Médico Ocupacionales y Guias de Diagnóstico de los Exámenes Médicos obligatorios por Actividad: RM No 312-2011/MINSA. Dirección General de Salud Ambiental, Perú; 2011.
2. Chaturvedi N, Cockcroft A. Tuberculosis screening in health service employees: who needs chest $X$ rays? Occup Med. 1992; 42(4):179-82.

3. Soto-Cabezas MG, Chávez-Pachas AM, ArrascoAlegre JC, Yagui-Moscoso MJA. Tuberculosis en trabajadores de salud en el Perú, 2013-2015. Rev Peru Med Exp Salud Publica. 2016; 33(4):607-15.

4. Consejo Nacional del Residentado Médico, CONAREME. Encuesta descriptiva: Percepción sobre el proceso de formación de médicos residentes en el sistema nacional de residentado médico (SINAREME) del Perú, 2016.

5. Ticona E, Huaman MA, Huaroto LM, Burgos M, Brett M, Escombe R, et al. Tuberculosis screening using ability to provide sputum in an endemic emergency department. Eur Respir J. 2016; 47(1):330-3. 